\title{
Measure Method of Submarine Pressure Hull Radial Initial Deflection Influenced By Different Selection of Common Point
}

\author{
PENG Fei ${ }^{1, a}$, LIU Xuchen ${ }^{1}$, ZHU Zhijie ${ }^{1}$, WANG Zhong ${ }^{1}$ \\ ${ }^{1}$ Department of Naval Architecture \& Ocean Engineering, Naval University of Engineering, Wuhan Hubei 430033, China
}

\begin{abstract}
While using Electronic Total Station to measure the deflection of the pressure hull, the measurement error was influenced by different selection of common points. To solve this problem, the coordinate values of pressure hull profiles were established at two different coordinate measuring stations. Selecting different position and number of common points to solve the analog circle initial deflection, we obtained the variation of the initial deflection calculation affected by analyzing the initial deflection and middle error of the measure point. The Simulation results show that the common point should be evenly distributed on the pressure hull, and the number of common points should be controlled at 4-7.

Keywords: coordinate transformation; common points; calculation accuracy; pressure hull radial initial deflectio.
\end{abstract}

\section{INTRODUCTION}

During the Submarine construction, controlling the initial deflection of the pressure hull is an important part, the current domestic pressure hull measuring method is usually using strut[1,2]. Measurement results of the strut method recorded by hand, it exist inefficient, large deviation, susceptible to human factors and other shortcomings. This method is difficult to achieve the construction requirements of the modern submarine, and the measurement reliability has not been verified. China has already begun the study about using the total station to measure the pressure hull initial deflection $[3,4]$, by using ETC to collect pressure hull profile measuring point data, after the plane fitting and circle fitting, fitted circle is calculated. Comparing fitted circle and measuring point data, the initial deflection results could be get. But in the measurement process, there are vertical and horizontal bulkhead barriering inside the submarine, all the measuring point data could not be got in one station, moving the total station is needed, namely transfer station, then we can complete

the measurement of the pressure hull profile. By measuring common points of different stations and calculating conversion parameters, we could complete the conversion of space coordinate system. Selecting the common points position affect the accuracy of the coordinate transformation, and also affect the calculation results of pressure hull radial initial deflection.

In this paper, we simulate two different sets of coordinates of 32 measuring points in the submarine pressure profiles. The circle is divided into two parts. By selecting different points as common points, we calculate coordinate transformation parameters, and then using the

\footnotetext{
a Corresponding author: liuxc2115@163.com
}

parameters transform all the measuring point to one coordinate system, the initial deflection could be obtained. We put the initial deflection and middle error which is used to judge the average errors of the measuring point, reflect the degree of influence of different common point selection.

\section{THEORETICAL MODEL}

\subsection{SIMULATION DATA MODEL}

In order to simulate submarine pressure hull profile, we establish a standard circle, set 32 true point equally spaced along the circle. Then we add Gaussian noise at level $\sigma=1$ to each true point in $\mathrm{x}, \mathrm{y}, \mathrm{z}$ direction.

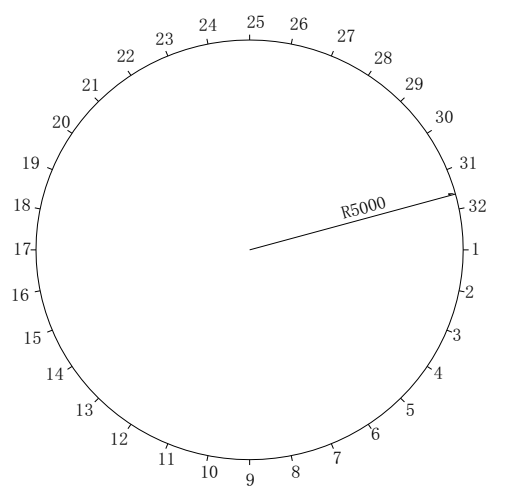

Fig. 1. Measuring point distribution of simulate circle

As figure 1 shows, we select different points for solving the coordinate transformation parameters, different positions of points constitute different 
combinations, the rest of the measuring points is used for inspection point to obtain the initial deflection value influences in different position.

\subsection{COORDINATE FORMULA}

TRANSFORMATION

Six-parameter rectangular coordinate transformation model [5]:

$$
\left[\begin{array}{c}
X^{\prime} \\
Y^{\prime} \\
Z^{\prime}
\end{array}\right]=\left[\begin{array}{c}
\Delta X \\
\Delta Y \\
\Delta Z
\end{array}\right]+R_{a}(f) R_{b}(w) R_{c}(k)\left[\begin{array}{c}
X \\
Y \\
Z
\end{array}\right]
$$

In equation (1), $\left[\begin{array}{lll}X^{\prime} & Y^{\prime} & Z^{\prime}\end{array}\right]^{T},\left[\begin{array}{lll}X & Y & Z\end{array}\right]$ represent coordinate value in the two different coordinate system. $\Delta X, \Delta Y, \Delta Z$, represent three translation parameters. $f, w, k$ represent three rotation parameters. $R_{a}(f)$, $R_{b}(w), R_{c}(k)$ denote rotation matrixes along the axis $\mathrm{x}$, $\mathrm{y}, \mathrm{z}$. We use residual equation

$$
\begin{gathered}
V=B d X-L \\
X=\left[\begin{array}{llllll}
\Delta X & \Delta Y & \Delta Z & f & w & k
\end{array}\right]^{T}
\end{gathered}
$$

The commonly used method for solving the nonlinear equations is Gauss-Newton iterative method. Iterative formula

$$
\begin{array}{ccr}
\text { is } & X^{(k+1)}=X^{(k)}+d X^{(k)} & \text { and } \\
\left.d X^{(\mathrm{k})}=B^{T}\left(X^{(k)}\right) B\left(X^{(k)}\right)\right)^{-1} B^{T}\left(X^{(k)}\right) L, & d X^{(k)} & \text { is the }
\end{array}
$$
correction matrix for coordinate transformation. Document [5] provide the specific method for calculating linear matrix B and constant matrix L. Set initial value for matrix $\mathrm{X}$ and put it into the residual equation. Cycle calculate until the modified value $d X$ less than the limit we set, then we can find out parameter matrix $\mathrm{X}$.

\subsection{PLANE FITTING PARAMETER ESTIMATION}

There are many ways from huge amounts of spatial point to fit planes, the most common ways are the least square method and characteristic method. The least square method assumes that the error exists only in the $\mathrm{Z}$ direction, but in actual engineering measurement, error exist in $\mathrm{x}, \mathrm{y}, \mathrm{z}$ directions. Therefore, the least square method is not suitable for our work.

Characteristic method expresses the plane as $a x+b y+c z=d$. It is natural to impose a constraint $a^{2}+b^{2}+c^{2}=1$. Despite the errors exist in any direction of the coordinate system, it could find the optimal plane parameters.

Let the measuring point data

$\left\{\left(x_{i}, y_{i}, z_{i}\right), i=1,2,3, \ldots, 32\right\}$ fed into the plane equation. We get the distance of measuring point to the plane.

$$
r_{i}=\left|a x_{i}+b y_{i}+c z_{i}-d\right|
$$

Taking $\sum r_{i}^{2}$ the minimum value of $\mathrm{A}$, we can get the best fitting plane. To solve the minimization problem one use a Lagrange multiplier $\lambda$ and reduce it to the function

$$
f=\sum_{i} r_{i}^{2}-\lambda\left(a^{2}+b^{2}+c^{2}-d\right)
$$

Take partial derivative of $f$ to form a characteristic equation

$$
\left[\begin{array}{lll}
\sum_{i} \Delta x_{i} \Delta x_{i} & \sum_{i} \Delta x_{i} \Delta y_{i} & \sum_{i} \Delta x_{i} \Delta z_{i} \\
\sum_{i} \Delta x_{i} \Delta y_{i} & \sum_{i} \Delta y_{i} \Delta y_{i} & \sum_{i} \Delta y_{i} \Delta z_{i} \\
\sum_{i} \Delta x_{i} \Delta z_{i} & \sum_{i} \Delta y_{i} \Delta z_{i} & \sum_{i} \Delta z_{i} \Delta z_{i}
\end{array}\right]\left[\begin{array}{l}
a \\
b \\
c
\end{array}\right]=\lambda\left[\begin{array}{l}
a \\
b \\
c
\end{array}\right]
$$

By solving the equation, we get the value of $a, b, c$.

\subsection{CIRCLE FITTING METHOD}

In this paper, the method for assess circular initial deflection is least square circle method [8]. We fit circle to the projection point. The value of initial defection is equal to the distance between the projection point and the fitting circle. According to the definition of the least square circle, we set the center of the circle as $(a, b, c)$, establish the function

$$
\begin{aligned}
F & =\sum\left(r_{i}^{2}-R^{2}\right)^{2} \\
& =\sum\left(x_{i}^{2}+y_{i}^{2}+z_{i}^{2}-2 a x_{i}-2 b y_{i}-2 c z_{i}+a^{2}+b^{2}+c^{2}-R^{2}\right)^{2}
\end{aligned}
$$

Take partial derivative of $F$ by using Lagrange multiplier method. It could return the maximum likelihood estimate of the circle parameters.

$$
\left(\hat{a}_{M L E}, \hat{b}_{M L E}, \hat{c}_{M L E}, \hat{R}_{M L E}\right)=\operatorname{agr} \min F(a, b, c, R)
$$

\section{EXAMPLE ANALYSIS}

We introduce middle error [9] as the standard of the measuring point offset. The middle error $\sigma=\sqrt{\frac{1}{n} \Delta^{T} \Delta}, n$ represent the observation number, $\Delta$ represent the observation error. As the true value of the simulation data have been given, it is easily to calculate the observation error. Then we use the initial deflection to reflect the degree of every point offset.

\subsection{THE COMMON POINTS OF CONTINUOUS DISTRIBUTION AND UNIFORM DISTRIBUTION}

Considering the distribution of common points may affect the transfer station measurement precision, we formulate the uniform distribution and continuous distribution of two kinds of scheme. 
Plan A: The common point of continuous distribution, select 1, 2, 3, and 4 points as the common points.

Plan B: The common point of uniform distribution, select $1,9,17$ and 25 points as the common points.

Use common points of the two pans to solve the transformation parameters:

Tab. 1. transformation parameters of the two plans

\begin{tabular}{ccc}
\hline parameter & $\begin{array}{c}\text { continuous distribution } \\
1,2,3,4\end{array}$ & $\begin{array}{c}\text { Uniform } \\
\text { distribution } \\
1,9,17,25\end{array}$ \\
\hline$\sigma$ & 40.5673186 & 2.18076965 \\
$\Delta X$ & 2975.33603 & 3000.06138 \\
$\Delta Y$ & 3991.17200 & 3998.96855 \\
$\Delta Z$ & 4977.46092 & 5001.17182 \\
$f$ & 0.51750838 & 0.52341012 \\
$w$ & 0.78346010 & 0.78533105 \\
$k$ & 1.04685711 & 1.04739621 \\
\hline
\end{tabular}

Table 1 shows the result of the different plans. Using transformation parameters to solve the remaining points, Initial deflection distribution line chart is obtained after the plane fitting and circle fitting.

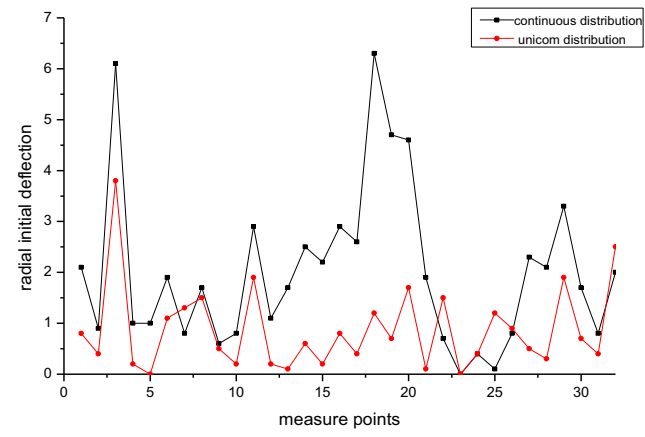

Fig. 2. The initial deflection curve of different common points distribution

As can be seen from Table 1, the parameters calculated by the two solutions vary widely. Comparing the middle error $\sigma\left(\sigma_{1}=40.56 \quad \sigma_{2}=2.18\right)$, the accuracy of the scheme 2 is far greater than the scheme 1 . It can be seen from Figure 2, when the common point continuous distribution, initial deflection solver result is very unstable, the greater the distance is, the common point of initial deflection fluctuate more significantly, the greater the error is. However, when the common point is uniformly distributed, the transformation accuracy of each measuring point is high, the initial deflection fluctuate in a small range. In summary, the distribution of the common points influent the initial deflection calculations, the actual measurement point should be evenly distributed in the pressure hull.

\subsection{THE NUMBER OF THE COMMON POINTS}

On the basis of uniform distribution of common point, we increase the number of common points. Then the transformation parameters are calculated, results are shown in Table 2 and results of initial deflection are shown in Figure 3. At last, the middle errors of the different common point are calculated, we can see the result in Figure 4.

Tab 2 the transformation parameters calculated by different number of common point

\begin{tabular}{|c|c|c|c|c|}
\hline $\begin{array}{l}\text { Paramete } \\
\mathrm{r}\end{array}$ & $\begin{array}{c}\text { Three } \\
\text { points } \\
(1,11,22)\end{array}$ & $\begin{array}{l}\text { Six points } \\
(1,6,11, \\
17,22,27)\end{array}$ & $\begin{array}{l}\text { Nine points } \\
(1,5,9,13 \text {, } \\
17,21,24 \text {, } \\
27,30)\end{array}$ & True value \\
\hline$\sigma$ & 3.19066304 & 2.54904418 & 2.332115 & 0 \\
\hline$\Delta X$ & $\begin{array}{c}2999.25889 \\
5\end{array}$ & $\begin{array}{c}3000.10313 \\
5\end{array}$ & 2999.54077 & 3000 \\
\hline$\Delta Y$ & $\begin{array}{c}4000.98693 \\
1\end{array}$ & $\begin{array}{c}3999.85968 \\
5\end{array}$ & $\begin{array}{c}4001.03013 \\
9\end{array}$ & 4000 \\
\hline$\Delta Z$ & $\begin{array}{c}5000.16828 \\
5\end{array}$ & $\begin{array}{c}4999.71325 \\
4\end{array}$ & $\begin{array}{c}4999.59492 \\
7\end{array}$ & 5000 \\
\hline$f$ & $\begin{array}{c}0.52323965 \\
9\end{array}$ & $\begin{array}{c}0.52363426 \\
6\end{array}$ & $\begin{array}{c}0.52353050 \\
5\end{array}$ & $\begin{array}{l}0.52359877 \\
6\end{array}$ \\
\hline$w$ & $\begin{array}{c}0.78501203 \\
1\end{array}$ & $\begin{array}{c}0.78526627 \\
8\end{array}$ & $\begin{array}{c}0.78522043 \\
9\end{array}$ & 0.785398 \\
\hline$k$ & $\begin{array}{c}1.04770587 \\
2\end{array}$ & $\begin{array}{c}1.04740215 \\
7\end{array}$ & $\begin{array}{c}1.04734461 \\
5\end{array}$ & 1.28661619 \\
\hline
\end{tabular}

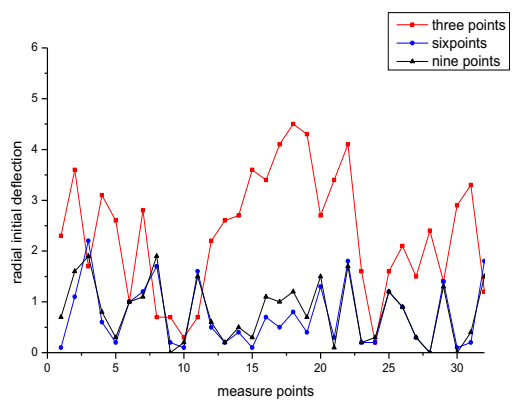

Fig. 3. The initial deflection curve of different number common points

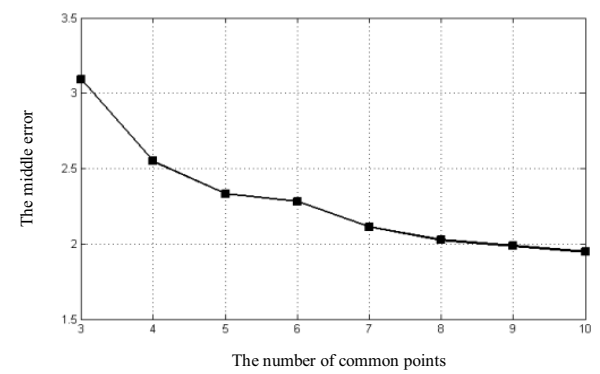

Fig. 4. The middle error curve of different number of common point

The data in Table 2 shows that the greater the selected numbers of the common points is, the higher the accuracy is. The results of the transformation parameters solvers tend to the true value. As can be seen from Figure 3 , selecting three common points, the curve of the initial deflection value fluctuate widely. When the numbers of the common points is increase to 6 and 9, fluctuation range of the initial deflection value decreases obviously and tend to converge. It can be seen in the middle error curve of the different number of common points (Figure 4 ), with the increase of the number of common points, the 
coordinate transformation accuracy tend to be higher. When the common points number increase from 3 to 4 , the precision can be improved a lot. When the common points selected in the seven or more, with the increase in the number of the common points, a slight increase is in conversion accuracy.

\section{CONCLUSION}

Common points should be evenly distributed on the pressure hull, the number of common points should be controlled at 4-7. With the increase of the point number, the accuracy of transfer station public will increase. However when we select more than seven points, it will be a little success to help improve the accuracy. There are complex structures in some cabin cross-section of submarine, selecting too many common points is not only difficult to arrange, but will increase the measurement workload.

\section{References}

1. Q. Guilin, The submarine construction technology, Beijing: National Defence Industry Press, 1982.

2. C. Lei. Naval construction process, Beijing: Haicao Press, 2003.
3. Z. Xiaojun and W. Peng, "Experimental study on submarine pressure-hull structure radial initial deflection calculate method", Ship Science and Technology, vol. 35(1), pp. 42-45, 2013.

4. W. Peng and Z. Xiaojun, "Investigation of Submarine Pressure hull Radial Flesibility Assess Method", Journal of Wuhan University of Technology (Transportation Science \&Engineering), vol. 37(1), pp. 149-152, 2013.

5. Z. Wenxian and T. benzao, "Non-linear adjustmentmodel of three-dimensional coordinate transformation", Geomatics and Information Science of Wuhan University, vol. 28(5), pp. 136-139, 2003,

6. W. Xinzhou, Theory and application of parameter estimation in nonlinear model, Wuhan: Wuhan University Press, 2002.

7. G. Yunlan and C. Xiaojun, "A Robust Method for Fitting a Plane to Point Clouds", Journal Of Tongji University( NatuRal Science), vol.36(7), pp. 981-985, 2008.

8. S. Gass and C. Witzgall. "On an approximate minimax circle closet to a set of point", Computers \& Operations Reasearch, vol. 31.pp. 637-643, 2004.

9. Z. Yueyin and P. Guorong, "Accuracy Of Coordinate Transfer Influenced By Different Distributions Of Common Points", Journal Of Geodesy And Geodynamics, vol. 33(2), pp. 105-109, 2013. 